\title{
Genetic Analysis for Grain Yield and Some Agronomic Traits in Some New White Maize Inbred Lines by Using Line X Tester Analysis
}

\author{
H.A.A. Gamea \\ Maize Research Section, Field Crops Research Institute, ARC, Egypt.
}

\begin{abstract}
Nineteen new white maize inbred lines were top crossed with two testers i.e.; Sd. 7 and Sd. 63 at Gemmeiza Research Station in 2017 growing season. The resulted 38 crosses in addition to three commercial check hybrids i.e. SC10, SC128 and SC 2031 were evaluated at Gemmeiza and Sids Agriculture Research Station Farms during 2018 growing season. Data were recorded for the following traits: days to 50\% silking, plant height, ear height and grain yield. Analysis of variance of crosses and their components, lines, testers and lines $\mathrm{x}$ testers showed highly significant differences for all traits, except lines $\mathrm{x}$ testers for days to $50 \%$ silking. $\sigma^{2} \mathrm{GCA}$ was larger than $\sigma^{2} \mathrm{SCA}$ for all traits. These results indicated the dominance of additive gene action in the inheritance of these traits. The inbred lines $\mathrm{L}_{4}, \mathrm{~L}_{7}, \mathrm{~L}_{10}$ and $\mathrm{L}_{19}$ (for earliness) and the inbred lines $\mathrm{L}_{1}, \mathrm{~L}_{5}, \mathrm{~L}_{10}, \mathrm{~L}_{11}$ and $\mathrm{L}_{12}$ (for grain yield) had high general combining ability. The crosses $\mathrm{L}_{10} \times \mathrm{T}_{1}$ (42.91 ard. /fed.), $\mathrm{L}_{11} \times \mathrm{T}_{1}$ (38.40 ard./fed.) and $\mathrm{L}_{14} \times \mathrm{T}_{1}(35.65 \mathrm{ard} / \mathrm{fed})$ had high mean values and highly significant SCA effects for grain yield.
\end{abstract}

Key words: Maize, Zea mays L., line x tester, Combining ability, crosses.

\section{INTRODUCTION}

Maize is one of the most important economic cereal summer crops all over the world. It ranks the third after wheat and rice contributes a highly percentage of maize production to livestock and poultry. Also, it is a row material for several industrial products. It possesses one of the most studied genetic systems among cereals which have motivated a rich history of research into the genetics of various traits in maize. In fact maize has been subjected to extensive genetic studies than any other crop (Hallauer and Miranda, 1988). High productivity hybrids is an objectives of plant breeders, through evaluating lines according to general and specific combining abilities. The procedure of topcross suggested by Davis (1927) was used early to evaluate combining abilities. The theory of specific (SCA) and general (GCA) combining abilities established by Sprague and Tatum (1942) had been used broadly in improving of several economic plant species. Darrah et al. (1972) and Harnor et al. (1973) reported that the inbred lines tester have the advantage of sampling errors of genetic variability within the testers and greater genetic variation among test crosses. AlNaggar et al.(1997) suggested that inbred line testers can be effectively used for evaluation of both general and specific combining abilities. Alamine et al. (2006), Sedhom et al. (2007) and Abd ElMottalb et al. (2013) indicated the relative importance of G.C.A. variance in controlling the inheritance of yield, its component and some morphological traits, such as days to $50 \%$ silking, plant height and ear height. Many investigators sugggested that G.C.A. effects were relatively more important than S.C.A. effects in inbred lines (Shehata 1992, Mosa 1996 and EL-Zeir 1999). While, (Shehata and Dhawan 1975) found that the S.C.A. effectes were more important than G.C.A. effects in inheritance of grain yeild. Many investigators sugggested that non additive gene effects played an important role in the inheritance of grain yield and some other agronmic traits Aly 2013, Izhar and Chakroborty 2013 and Umer et al. 2014. While, Abd El- Mottalb et al. 2013 and Abdalla 2014 found that preponderance of additive gene action in controlling inheritance of grain yield and its components.

The aims of this study were to estimat combining abilities of inbred line for grain yield and other agronomic traits and identify superior crosses with improved yielding ability in a maize breeding program

\section{MATERIALS AND METHODS}

This study was conducted at the experimental fields of Gemmeiza and Sids Agricultural Research stations, ARC, Egypt during summer seasons of 2017 and 2018. Twenty one white maize inbred lines of were used nineteen newly were developed namely Gm 8, 52, 64, 147, 150, 187, 194, 204, 212, 291, 294, 300, 327, 332, 357, 385, 386, 387 and 389) were used as female lines and two released inbred lines (Sd.7and Sd. 63) were used as testers (males). In 2017 summer season, top crosses were made between the nineteen white inbreed lines and the two testers at Gemmeiza Agriculture Research Station (Table 1). 
Table 1: Source population, designation and symbols for nineteen white inbred lines and two white inbred testers.

\begin{tabular}{|c|c|c|}
\hline Source population liens, & designation & symbols \\
\hline Gemmeiza white pop. & Gm.8, 52, 64, 147, 150, 187, 194, 204, 212, 291, 294. & $\mathrm{~L}_{1}: \mathrm{L}_{11}$ \\
\hline Giza 2 & Gm. $300,327,332,357$. & $\mathrm{L}_{12}: \mathrm{L}_{15}$ \\
\hline Tuxpino & Gm. $385,386,387,389$. & $\mathrm{L}_{16}: \mathrm{L}_{19}$ \\
\hline \multicolumn{3}{|l|}{ Testers } \\
\hline A.E.D* & Sd. 7 & $\mathrm{~T}_{1}$ \\
\hline TEP\# $5 * *$ & Sd. 63 & $\mathrm{~T}_{2}$ \\
\hline
\end{tabular}

At both of Gemmeiza and Sids Agriculture Research Stations, a 38 cross hybrids and three check hybrids single cross (SC 10, SC 128 and SC 2031) were evaluated in 2018 summer season. A randomized complete block design with four replications was used. Plot size was one row, $6 \mathrm{~m}$ long and $0.8 \mathrm{~m}$ apart. Seeds were planted in hills evenly spaced at $0.25 \mathrm{~m}$ with two kernels-hills. Seedlings were thinned to one plant-hill after 21 days from planting. All cultural practices for maize production were applied as recommended. Analysis of variance was done over locations according to Steel and Torrie (1980). Bartlett test was used to test the homogeneity of error variances. The procedure of line $\mathrm{x}$ tester analysis according to Kempthorne (1957) was used for estimating general and specific combining ability effects. Data were recorded on the following characters on plot basis for number of days to $50 \%$ silking, plant height $(\mathrm{cm})$, ear height (cm) and grain yield, ardab/faddan (ard/fed) adjusted to $15.5 \%$ moisture content (one ardeb = $140 \mathrm{~kg})$.

\section{RESULTS AND DISCUSSION}

Analysis of variance

Analysis of variance for the four traits (days to $50 \%$ silking, plant height, ear height and grain yield) from combined data were presented in Table (2).
Data cleared that, locations were significantly different These results were in agreement with those reported by Abd EL-Azeem (2011), Ibrahim et al. (2012) EL- Gazzar, et al. (2013), Abdallah (2014), Gamea (2015) and Gamea et al. (2018). Analysis of variance of crosses and their portions lines, testers and lines $\mathrm{x}$ testers showed significant differences all traits, except lines $\mathrm{x}$ testers for days to $50 \%$ silking. These findings indicates that the inbred lines behaved differently in respective tester crosses. These results are in agreement with those obtained by, Mosa et al. (2008), Abd EL- Hadi et al. (2009), EL-Gazzar and Khalil (2012) and Aboyousef et al. (2016).

\section{Gene actions}

Genetic variance components for all studied traits over locations and their interaction with locations are shown in Table (3). Results revealed that estimates of $\left(\sigma^{2} \mathrm{GCA}(\mathrm{T})\right)$ were higher in magnitude than $\left(\sigma^{2} \operatorname{GCA}(\mathrm{L})\right)$ for plant height, ear height and grain yield, indicated that most of the total GCA variances were due to the testers. Also, general combining ability variance components, $\sigma^{2} \mathrm{GCA}$ was larger than $\sigma^{2} \mathrm{SCA}$ for all traits. These results indicated the dominance of additive over non-additive gene action in the inheritance of these traits.

Table 2: Analysis of variance for days to $50 \%$ silking, plant height, ear height and grain yield traits of 38 top crosses combined across two locations (2018).

\begin{tabular}{llcccc}
\hline S. O. V & Df & $\begin{array}{c}\text { Days to 50\% } \\
\text { silking }\end{array}$ & $\begin{array}{c}\text { Plant height } \\
\mathbf{c m}\end{array}$ & $\begin{array}{c}\text { Ear height } \\
\mathbf{c m}\end{array}$ & $\begin{array}{c}\text { Grain yield } \\
\text { ard./fed. }\end{array}$ \\
\hline Loc. & 1 & $115.030^{* *}$ & $2968.750^{* *}$ & $3592.188^{* *}$ & $248.880^{* *}$ \\
\hline Rep/Loc. & 6 & $7.315^{* *}$ & $935.471^{* *}$ & $1358.745^{* *}$ & 10.044 \\
\hline Crosses (C) & 37 & $8.667^{* *}$ & $1471.915^{* *}$ & $1075.482^{* *}$ & $210.004^{* *}$ \\
\hline Lines (L) & 18 & $12.569^{* *}$ & $1324.041^{*}$ & $925.037^{* *}$ & $117.163^{* *}$ \\
\hline Testers (T) & 1 & $34.898^{* *}$ & $19745.066^{* *}$ & $15848.766^{* *}$ & $4215.695^{* *}$ \\
\hline L x T & 18 & 3.308 & $604.614^{* *}$ & $405.190^{* *}$ & $80.307^{* *}$ \\
\hline C/Loc. & 37 & $3.786^{*}$ & $424.662^{* *}$ & $304.181^{* *}$ & $25.188^{* *}$ \\
\hline L X Loc. & 18 & $3.412^{*}$ & $287.674 * *$ & $386.458^{* *}$ & $21.126^{*}$ \\
\hline T X Loc. & 1 & $27.240^{* *}$ & $636.842^{* *}$ & $742.188^{* *}$ & $66.728^{* *}$ \\
\hline L x T x Loc. & 18 & 2.858 & $549.863^{* *}$ & $197.569 *$ & $26.942^{* *}$ \\
\hline Pooled error & 222 & 2.042 & 107.600 & 113.699 & 10.907 \\
\hline CV \% & & 2.3 & 4.3 & 8.0 & 11.6 \\
\hline
\end{tabular}

*, ** Indicating significant at 0.05 and 0.01 levels of probability, respectively. 
Table 3: Estimates of variance component for days to $50 \%$ silking, plant height, ear height and grain yield traits

\begin{tabular}{lcccc}
\hline S.O.V & $\begin{array}{c}\text { Days to 50\% } \\
\text { Silking }\end{array}$ & $\begin{array}{c}\text { Plant height } \\
\text { cm }\end{array}$ & $\begin{array}{c}\text { Ear height } \\
\text { cm }\end{array}$ & $\begin{array}{c}\text { Grain yield } \\
\text { ard./fed. }\end{array}$ \\
\hline$\sigma^{\mathbf{2} \text { GCA-L }}$ & 0.54 & 61.35 & 20.68 & 2.67 \\
\hline$\sigma^{\mathbf{2}}$ GCA-T & 0.05 & 125.35 & 98.02 & 26.94 \\
\hline$\sigma^{\mathbf{2}}$ GCA(average) & 0.74 & 167.29 & 140.16 & 19.06 \\
\hline$\sigma^{2}$ SCA & 0.06 & 6.84 & 25.95 & 6.67 \\
\hline$\sigma^{\mathbf{2}}$ GCAL x Loc. & 0.07 & -32.77 & 23.61 & -0.73 \\
\hline$\sigma^{2}$ GCAT x Loc. & 0.32 & 1.14 & 7.17 & 0.52 \\
\hline$\sigma^{2}$ GCA x Loc. & 0.07 & -32.77 & 23.61 & -0.73 \\
\hline$\sigma^{2}$ SCA x Loc. & 0.20 & 110.57 & 20.97 & 4.01 \\
\hline All
\end{tabular}

All negative estimates of variance were considered zero.

Similar results have been reported in maize Aly (2004), Kumar et al.(2005). Abd El-Mottalb (2014) and Gamea (2015). Variance of $\sigma^{2} \mathrm{SCA} \times$ locations interaction was higher than the variance of $\sigma^{2} \mathrm{GCA} \times$ locations for most studied traits (days to $50 \%$ sillking, plant height and grain yield). These results indicated that non additive type of gene action was more affected by environmental conditions. Similar results were reported, Attia et al. (2015), AbdelMoneam et al. (2014), Darwich et al. (2016) and Abu shosha and Habouh (2019).

\section{The proportional contribution}

The contribution of lines, testers and their interactions to total variances are presented in Table 4. The proportional contribution of line to total variances was much higher than tester and line $\mathrm{x}$ tester in all traits, except for grain yield, where,s the contribution of tester was higher than the lines and lines $\mathrm{x}$ testers. These suggest female parent maximum contributed to total variance. Amin et al. (2014). Mean performance, GCA and SCA effects and superiority percentage for all traits under this study are presented from Table 5 to Table 8.

Days to 50\% silking:

Data in (Table 5) cleared that, means of days to $50 \%$ silking ranged from 59.6 days $\left(\mathrm{L}_{10} \times \mathrm{T}_{1}\right)$ to $63.8\left(\mathrm{~L}_{16} \times \mathrm{T}_{1}\right)$ and ranged from 60.9 (SC 128) to 64.4 (SC 2031) for crosses and checks, respectively. In the same time, side 4 crosses had lower values in earlines compared to the early check SC 128 . The inbred lines; $\mathrm{L}_{4}, \mathrm{~L}_{7}$ and $\mathrm{L}_{10}$ exhibited highly significant GCA effects towards earlines, while the tester $\left(\mathrm{T}_{2}\right)$ was a good tester of highly significant
GCA effects for days to $50 \%$ silking. Non of the crosses had a significant SCA effects and superiority percentage relative to short check (s.c128) for that trait.

\section{Plant height:}

Regarding the results in (Table 6) values of plant height showed that twelve crosses had low values of plant height, four $\left(\mathrm{L}_{3} \times \mathrm{T}_{2}\right),\left(\mathrm{L}_{6} \times \mathrm{T}_{2}\right)$, ( $\mathrm{L}_{7}$

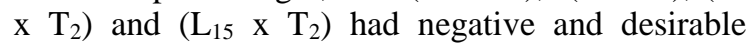
significant short plant height compared the short check (s.c128). Five inbred line $\left(\mathrm{L}_{2}, \mathrm{~L}_{3}, \mathrm{~L}_{13}\right.$ and $\left.\mathrm{L}_{15}\right)$ and tester $\left(\mathrm{T}_{2}\right)$ had negative and desirable significant of GCA effects for this trait. On the other hand Four crosses; $\left(\mathrm{L}_{2} \times \mathrm{T}_{1}\right),\left(\mathrm{L}_{7} \times \mathrm{T}_{2}\right),\left(\mathrm{L}_{11} \times \mathrm{T}_{2}\right)$ and $\left(\mathrm{L}_{12} \times \mathrm{T}_{1}\right)$ had negative and desirable significant SCA effects. Four crosses $\left(\mathrm{L}_{3} \times \mathrm{T}_{2}\right),\left(\mathrm{L}_{6} \times \mathrm{T}_{2}\right),\left(\mathrm{L}_{7} \times \mathrm{T}_{2}\right)$ and $\left(\mathrm{L}_{15} \mathrm{x}\right.$ $\mathrm{T}_{2}$ ) exhibited desirable and highly significant superiority percentage for shortness relative to short check SC 128.

\section{Ear height:}

For ear height data in (table 7) cleared that, out of the 38 test crosses, four test crosses; $\left(\mathrm{L}_{3} \times \mathrm{T}_{2}\right)$, $\left(\begin{array}{lllll}\mathrm{L}_{7} \mathrm{X} & \mathrm{T}_{2}\end{array}\right),\left(\begin{array}{llll}\mathrm{L}_{13} & \mathrm{x} & \mathrm{T}_{2}\end{array}\right)$ and $\left(\mathrm{L}_{15} \times \mathrm{T}_{2}\right)$ exhibited desirable and highly significant means compared to the best check SC 128 for low ear placement. The inbred lines; $\mathrm{L}_{2}, \mathrm{~L}_{3}$ and $\mathrm{L}_{13}$ and tester; $\mathrm{T} 2$ exhibited negative and desirable significant of GCA effects for ear height. Eight crosses $\left(\mathrm{L}_{2} \mathrm{xT}_{1}\right),\left(\mathrm{L}_{7} \times \mathrm{T}_{2}\right),\left(\mathrm{L}_{12}\right.$ $\left.\mathrm{x} \mathrm{T}_{1}\right),\left(\mathrm{L}_{18} \times \mathrm{T}_{1}\right),\left(\mathrm{L}_{3} \times \mathrm{T}_{2}\right),\left(\mathrm{L}_{7} \times \mathrm{T}_{2}\right),\left(\mathrm{L}_{13} \times \mathrm{T} 2\right)$ and $\left(\begin{array}{lll}\mathrm{L}_{15} & \mathrm{x} & \mathrm{T}_{2}\end{array}\right)$ had negative and desirable highly significant of SCA effects and superiority percentage relative the best check SC 128 for low ear placement, respectively.

Table 4: Proportional contribution of line, tester and their interaction to total variance.

\begin{tabular}{lcccc}
\hline Source & $\begin{array}{c}\text { Days to 50\% } \\
\text { silking }\end{array}$ & $\begin{array}{c}\text { Plant height } \\
\text { cm }\end{array}$ & $\begin{array}{c}\text { Ear height } \\
\text { cm }\end{array}$ & $\begin{array}{c}\text { Grain yield } \\
\text { ard./fed. }\end{array}$ \\
\hline Due to line & 70.6 & 43.8 & 41.8 & 27.1 \\
\hline Due to tester & 10.9 & 36.3 & 39.8 & 54.3 \\
\hline Due to line $x$ tester & 18.6 & 20.0 & 18.3 & 18.6 \\
\hline
\end{tabular}


Table 5: Mean performance, combining ability effects and superiority percentage for days to 50\% silking.

\begin{tabular}{|c|c|c|c|c|c|c|c|c|c|c|c|}
\hline \multirow[t]{2}{*}{ Lines } & \multicolumn{3}{|c|}{ Means (day) } & \multicolumn{3}{|c|}{ GCA effects } & \multicolumn{3}{|c|}{ SCA effects } & \multicolumn{2}{|c|}{$\begin{array}{l}\text { Superiority percentage } \\
\text { relative to early check } \\
\text { SC } 128 \text { (60.9day) }\end{array}$} \\
\hline & & 1 & $\mathbf{T}_{2}$ & \multicolumn{3}{|c|}{ Lines } & \multicolumn{2}{|c|}{$\mathbf{T}_{1}$} & $\mathbf{T}_{2}$ & $\mathbf{T}_{1}$ & $\mathbf{T}_{2}$ \\
\hline $\mathrm{L}_{1}$ & & .3 & 61.6 & \multicolumn{3}{|c|}{0.4} & \multicolumn{2}{|c|}{0.0} & 0.0 & $2.3^{*}$ & 1.2 \\
\hline $\mathrm{L}_{2}$ & & .1 & 60.6 & \multicolumn{3}{|c|}{-0.2} & \multicolumn{2}{|c|}{0.4} & -0.4 & 2.1 & -0.4 \\
\hline $\mathrm{L}_{3}$ & & .3 & 61.5 & \multicolumn{3}{|c|}{0.3} & \multicolumn{2}{|c|}{0.0} & 0.0 & $2.3^{*}$ & 1.0 \\
\hline $\mathrm{L}_{4}$ & & .3 & 59.9 & \multicolumn{3}{|c|}{$-1.0 * *$} & \multicolumn{2}{|c|}{0.3} & -0.3 & 0.6 & -1.6 \\
\hline $\mathrm{L}_{5}$ & & .8 & 61.5 & \multicolumn{3}{|c|}{0.6} & \multicolumn{2}{|c|}{0.3} & -0.3 & $3.1 * *$ & 1.0 \\
\hline L6 & & .0 & 61.8 & \multicolumn{3}{|c|}{-0.2} & \multicolumn{2}{|c|}{-0.7} & 0.7 & 0.2 & 1.4 \\
\hline $\mathrm{L}_{7}$ & & .5 & 59.9 & \multicolumn{3}{|c|}{$-0.9 * *$} & \multicolumn{2}{|c|}{0.5} & -0.5 & 1.0 & -1.6 \\
\hline $\mathrm{L}_{8}$ & & .9 & 61.6 & \multicolumn{3}{|c|}{0.2} & \multicolumn{2}{|c|}{-0.2} & 0.2 & 1.6 & 1.2 \\
\hline $\mathrm{L}_{9}$ & & & 60.5 & \multicolumn{3}{|c|}{-0.4} & \multicolumn{2}{|c|}{0.3} & -0.3 & 1.4 & -0.6 \\
\hline $\mathrm{L}_{10}$ & & .6 & 60.1 & \multicolumn{3}{|c|}{$-1.7 * *$} & \multicolumn{2}{|c|}{-0.6} & 0.6 & -2.1 & -1.2 \\
\hline $\mathrm{L}_{11}$ & & .9 & 61.4 & & & & -0 & & 0.6 & 0.0 & 0.8 \\
\hline $\mathrm{L}_{12}$ & & & 60.5 & & & & 0 . & & -0.6 & 2.5 & -0.6 \\
\hline $\mathrm{L}_{13}$ & & & 62.4 & & & & -0 & & 0.9 & 0.6 & $2.5 * *$ \\
\hline $\mathrm{L}_{14}$ & & & 62.9 & & & & -0 & & 0.3 & $3.5 * *$ & $3.3 * *$ \\
\hline $\mathrm{L}_{15}$ & & & 60.0 & & & & 0. & & -0.7 & 1.8 & -1.4 \\
\hline $\mathrm{L}_{16}$ & & & 63.3 & & & & -0 & & 0.1 & $4.7 * *$ & $3.9 * *$ \\
\hline $\mathrm{L}_{17}$ & & & 62.4 & & & & 0 . & & -0.1 & $3.9 * *$ & $2.5^{*}$ \\
\hline $\mathrm{L}_{18}$ & & & 61.4 & & & & -0 & & 0.1 & 1.6 & 0.8 \\
\hline $\mathrm{L}_{19}$ & & .3 & 60.0 & & & & 0. & & -0.3 & 0.6 & -1.4 \\
\hline & $\mathrm{SC} 10$ & & 64.1 & LSD & $5 \%$ & 0.7 & LSD & $5 \%$ & 1.0 & $5 \%$ & 1.4 \\
\hline & SC 1 & & 60.9 & $\mathrm{~g}_{\mathrm{i}}$ & $1 \%$ & 0.9 & $\mathrm{~s}_{\mathrm{ij}}$ & $1 \%$ & 1.3 & $1 \%$ & 1.8 \\
\hline & SC 2 & & 64.4 & LSD & $5 \%$ & 1.0 & LSD & $5 \%$ & 1.4 & & \\
\hline & I SD & $5 \%$ & 1.4 & $g_{i}-g_{j}$ & $1 \%$ & 1.3 & $\mathrm{~S}_{\mathrm{ij}} \mathrm{S}_{\mathrm{ki}}$ & $1 \%$ & 1.8 & & \\
\hline & LSD & $1 \%$ & 1.8 & Tester & & & & & & & \\
\hline & & & & & $\Gamma 1$ & 0.3 & & & & & \\
\hline & & & & & $\Gamma 2$ & $-0.3^{* *}$ & & & & & \\
\hline & & & & LSD & $5 \%$ & 0.2 & & & & & \\
\hline & & & & $\mathrm{g}_{\mathrm{i}}$ & $1 \%$ & 0.3 & & & & & \\
\hline & & & & LSD & $5 \%$ & 0.3 & & & & & \\
\hline & & & & $\mathrm{g}_{\mathrm{i}} \mathrm{g}_{\mathrm{j}}$ & $1 \%$ & 0.4 & & & & & \\
\hline
\end{tabular}

*,** Indicating significant at 0.05 and 0.01 levels of probability, respectively.

\section{Grain yield (ard./fed.):}

The main objective of maize breeders is to develop a high yielding maize hybrids. The mean performance of grain yield ranged from 19.93 to 42.91 for crosses and from 27.78 to 33.87 for checks, respectively (Table 8$)$. Six crosses $\left(\mathrm{L}_{1} \mathrm{x} \mathrm{T}_{1}\right.$, $\mathrm{L}_{7} \times \mathrm{T}_{1}, \mathrm{~L}_{10} \times \mathrm{T}_{1}, \mathrm{~L}_{11} \times \mathrm{T}_{1}, \mathrm{~L}_{12} \times \mathrm{T}_{1}$ and $\mathrm{L}_{14} \times \mathrm{T}_{1}$ ), nine crosses $\left(\left(\mathrm{L}_{1} \times \mathrm{T}_{1}, \mathrm{~L}_{4} \times \mathrm{T}_{1}, \mathrm{~L}_{5} \times \mathrm{T} 1, \mathrm{~L}_{7} \times \mathrm{T}_{1}, \mathrm{~L}_{8} \mathrm{x}\right.\right.$ $\mathrm{T}_{1}, \mathrm{~L}_{10} \times \mathrm{T}_{1}, \mathrm{~L}_{11} \times \mathrm{T}_{1}, \mathrm{~L}_{12} \times \mathrm{T}_{1}$ and $\left.\mathrm{L}_{14} \times \mathrm{T}_{1}\right)$ and two crosses $\left(\mathrm{L}_{10} \times \mathrm{T}_{1}\right.$ and $\left.\mathrm{L}_{11} \mathrm{x} \mathrm{T}_{1}\right)$ had significantly mean values of yield better than the checks SC 10,
SC 128 and SC 2031, respectively. Regarding data in Table (8), five inbred line; $\mathrm{L}_{1}, \mathrm{~L}_{5}, \mathrm{~L}_{10}, \mathrm{~L}_{11}$ and $\mathrm{L}_{12}$ had positive and highly significant of GCA effects. From the testers, T1 (SD 7) had positive and highly significant GCA effects for this trait. Five crosses ( $\mathrm{L}_{10} \mathrm{X} \mathrm{T}_{1}, \mathrm{~L}_{11} \times \mathrm{T}_{1}, \mathrm{~L}_{14} \times \mathrm{T}_{1}, \mathrm{~L}_{16} \mathrm{X} \mathrm{T}_{2}$ and $\mathrm{L}_{17} \times \mathrm{T}_{1}$ ) exhibited significant and highly significant SCA effects for grain yield. The superiority percentage relative to the best checks (SC 2031) ranged from $41.14 \%$ for crosses L13x T2 to $26.71 \%$ for crosses $\mathrm{L}_{10} \mathrm{X} \mathrm{T}_{1}$ and two crosses; $\mathrm{L}_{10} \mathrm{x} \mathrm{T}_{1}$ and $\mathrm{L}_{11} \times \mathrm{T}_{1}$ had 
positive and highly significant superiority percentage for grain yield.

Repenting on the previous might be concludes that, the inbred lines $\mathrm{L}_{4}, \mathrm{~L}_{7}, \mathrm{~L}_{10}$ and $\mathrm{L}_{19}$ are good donor for earlinees and the inbred lines $\mathrm{L}_{1}, \mathrm{~L}_{5}, \mathrm{~L}_{10}$, $\mathrm{L}_{11}$ and $\mathrm{L}_{12}$ that had high general combining ability. For grain yield might be utilized in the national hybrid breeding programs to improve such traits.
Also, these lines have high potential to transfer these desirable characters to their cross progenies. Crosses $\mathrm{L}_{10} \mathrm{x} \mathrm{T}_{1}$ (42.91 ard. /fed.), $\mathrm{L}_{11} \times \mathrm{T}_{1}$ (38.40 ard. /fed.) and $\mathrm{L}_{14} \times \mathrm{T}_{1}(35.65$ ard. /fed.) had high means values and highly significant SCA effects for grain yield, so might be used in maize hybrid development programs.

Table 6: Mean performance, combining ability effects and superiority percentage for plant height.

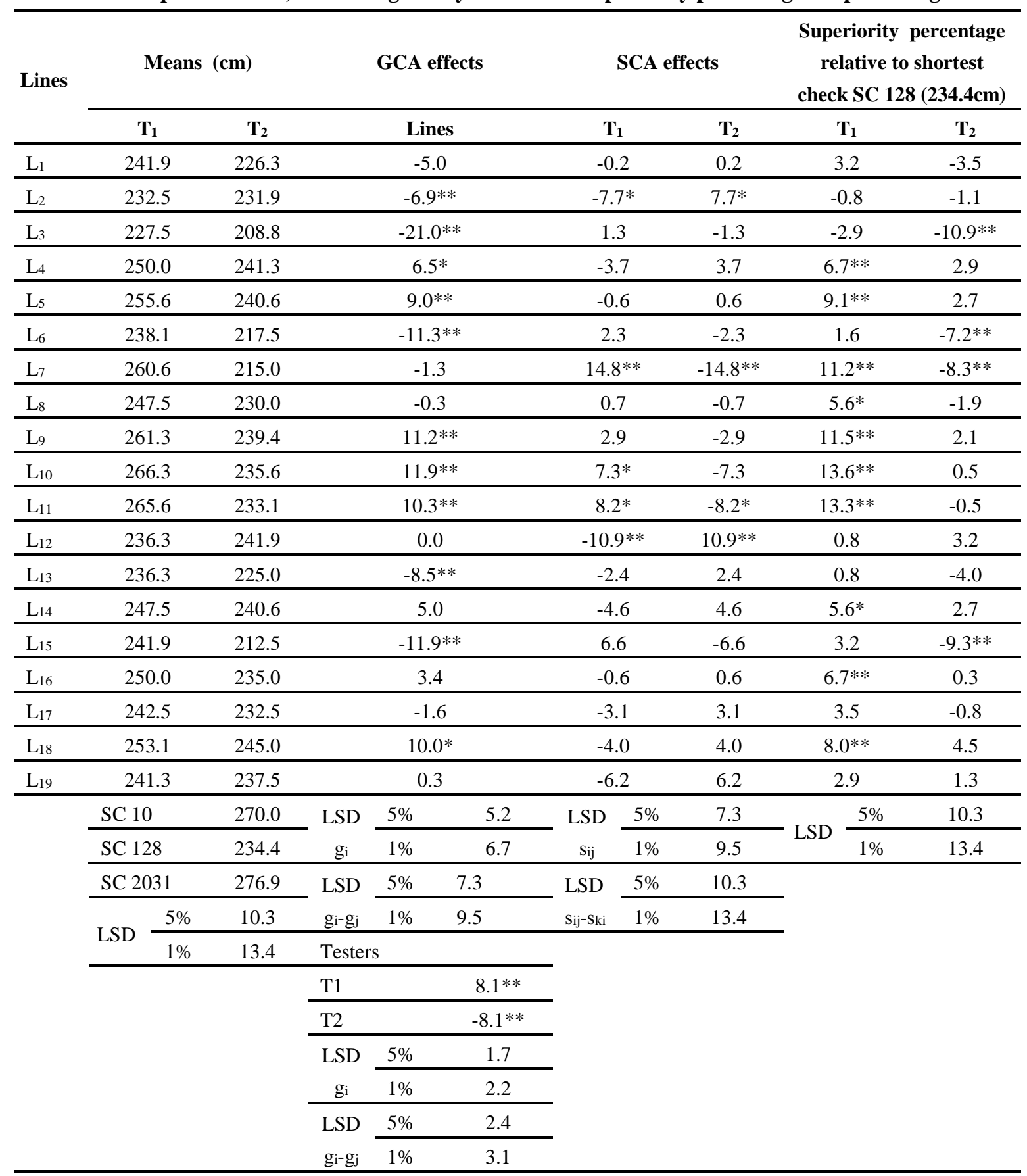

\footnotetext{
*, ** Indicating significant at 0.05 and 0.01 levels of probability, respectively.
} 
Table 7: Mean performance, combining ability effects and superiority percentage for ear height.

\begin{tabular}{|c|c|c|c|c|c|c|c|c|c|c|c|c|}
\hline \multirow[t]{2}{*}{ Lines } & \multicolumn{3}{|c|}{ Means (cm) } & \multicolumn{3}{|c|}{ GCA effects } & \multicolumn{3}{|c|}{ SCA effects } & \multicolumn{3}{|c|}{$\begin{array}{c}\text { Superioritypercentage } \\
\text { relative to lowest ear } \\
\text { placement check SC } 128 \\
(131.3 \mathrm{~cm}) \\
\end{array}$} \\
\hline & \multicolumn{2}{|c|}{$\mathbf{T}_{\mathbf{1}}$} & $\mathbf{T}_{2}$ & \multicolumn{3}{|c|}{ Lines } & $\mathbf{T}_{1}$ & \multicolumn{2}{|c|}{$\mathbf{T}_{2}$} & \multicolumn{2}{|c|}{$\mathbf{T}_{1}$} & $\mathbf{T}_{2}$ \\
\hline $\mathrm{L}_{1}$ & \multicolumn{2}{|c|}{135.0} & 127.5 & \multicolumn{3}{|c|}{-2.0} & -3.5 & \multicolumn{2}{|c|}{3.5} & \multicolumn{2}{|c|}{2.9} & -2.9 \\
\hline $\mathrm{L}_{2}$ & \multicolumn{2}{|c|}{124.4} & 127.5 & \multicolumn{3}{|c|}{$-7.3 * *$} & $-8.8^{*}$ & \multicolumn{2}{|c|}{$8.8^{*}$} & \multicolumn{2}{|c|}{-5.2} & -2.9 \\
\hline $\mathrm{L}_{3}$ & \multicolumn{2}{|c|}{121.3} & 108.1 & \multicolumn{3}{|c|}{$-18.6 * *$} & -0.7 & \multicolumn{2}{|c|}{0.7} & \multicolumn{2}{|c|}{-7.6} & $-17.6^{* *}$ \\
\hline $\mathrm{L}_{4}$ & \multicolumn{2}{|c|}{145.0} & 128.8 & \multicolumn{3}{|c|}{3.6} & 0.9 & \multicolumn{2}{|c|}{-0.9} & \multicolumn{2}{|c|}{$10.5 * *$} & -1.9 \\
\hline $\mathrm{L}_{5}$ & \multicolumn{2}{|c|}{151.9} & 137.5 & \multicolumn{3}{|c|}{$11.4 * *$} & 0.0 & & .0 & & $7 * *$ & 4.8 \\
\hline L6 & 14 & 0.6 & 122.5 & & -1.7 & & 1.8 & & .8 & & .1 & -6.7 \\
\hline $\mathrm{L}_{7}$ & 15 & 0.0 & 111.3 & & -2.6 & & $12.2 * *$ & & $2 * *$ & & $3 * *$ & $-15.2^{* *}$ \\
\hline $\mathrm{L}_{8}$ & 14 & 4.4 & 127.5 & & 2.7 & & 1.2 & & .2 & & $.0 *$ & -2.9 \\
\hline $\mathrm{L}_{9}$ & 14 & 5.0 & 128.8 & & 3.6 & & 0.9 & & .9 & & $5 * *$ & -1.9 \\
\hline $\mathrm{L}_{10}$ & 15 & 5.6 & 130.0 & & $9.6^{*}$ & & 5.6 & & 5.6 & & $6 * *$ & -1.0 \\
\hline $\mathrm{L}_{11}$ & 14 & 6.9 & 125.6 & & 3.0 & & 3.4 & & 3.4 & & $9 * *$ & -4.3 \\
\hline $\mathrm{L}_{12}$ & 13 & 7.5 & 138.1 & & 4.6 & & $-7.5^{*}$ & & $5 *$ & & .8 & 5.2 \\
\hline $\mathrm{L}_{13}$ & 13 & 0.6 & 108.1 & & $-13.9^{\prime}$ & & 4.0 & & 1.0 & & .5 & $-17.6 * *$ \\
\hline $\mathrm{L}_{14}$ & 14 & 3.8 & 130.6 & & 3.9 & & -0.7 & & .7 & & $5 *$ & -0.5 \\
\hline $\mathrm{L}_{15}$ & 13 & 7.5 & 118.8 & & -5.1 & & 2.2 & & 2.2 & & .8 & $-9.5^{*}$ \\
\hline $\mathrm{L}_{16}$ & 14 & 8.1 & 127.5 & & 4.6 & & 3.1 & & 3.1 & & $9 * *$ & -2.9 \\
\hline $\mathrm{L}_{17}$ & 13 & 3.8 & 125.6 & & -3.6 & & -3.2 & & .2 & & 9 & -4.3 \\
\hline $\mathrm{L}_{18}$ & 14 & 1.3 & 142.5 & & $8.6 *$ & & $-7.8 *$ & & $8 *$ & & .6 & $8.6^{*}$ \\
\hline $\mathrm{L}_{19}$ & 13 & 6.3 & 128.1 & & -1.1 & & -3.2 & & .2 & & .8 & -2.4 \\
\hline & SC 10 & & 157.5 & LSD & $5 \%$ & 5.3 & & $5 \%$ & 7.5 & & $5 \%$ & 10.6 \\
\hline & SC 12 & & 131.3 & $\mathrm{~g}_{\mathrm{i}}$ & $1 \%$ & 6.9 & $\operatorname{LSD~}_{\mathrm{ij}}$ & $1 \%$ & 9.7 & & $1 \%$ & 13.8 \\
\hline & SC 20 & & 157.5 & LSD & $5 \%$ & 7.5 & & $5 \%$ & 10.6 & & & \\
\hline & LSD & $5 \%$ & 10.6 & $\mathrm{~g}_{\mathrm{i}}-\mathrm{g}_{\mathrm{j}}$ & $1 \%$ & 9.7 & LSD $s_{\mathrm{ij}-} \mathrm{S}_{\mathrm{ki}}$ & $1 \%$ & 13.8 & & & \\
\hline & & $1 \%$ & 13.8 & Tester & & & & & & & & \\
\hline & & & & $\mathrm{T}$ & & $7.2 * *$ & & & & & & \\
\hline & & & & $\mathrm{T}$ & & $-7.2 * *$ & & & & & & \\
\hline & & & & LSD & $5 \%$ & 1.7 & & & & & & \\
\hline & & & & $\mathrm{g}_{\mathrm{i}}$ & $1 \%$ & 2.2 & & & & & & \\
\hline & & & & LSD & $5 \%$ & 2.4 & & & & & & \\
\hline & & & & $\mathrm{g}_{\mathrm{i}} \mathrm{g}_{\mathrm{j}}$ & $1 \%$ & 3.2 & & & & & & \\
\hline
\end{tabular}

\footnotetext{
*, ** Indicating significant at 0.05 and 0.01 levels of probability, respectively.
} 
Table 8: Mean performance, combining ability effects and superiority percentage for grain yield ard./ fed.

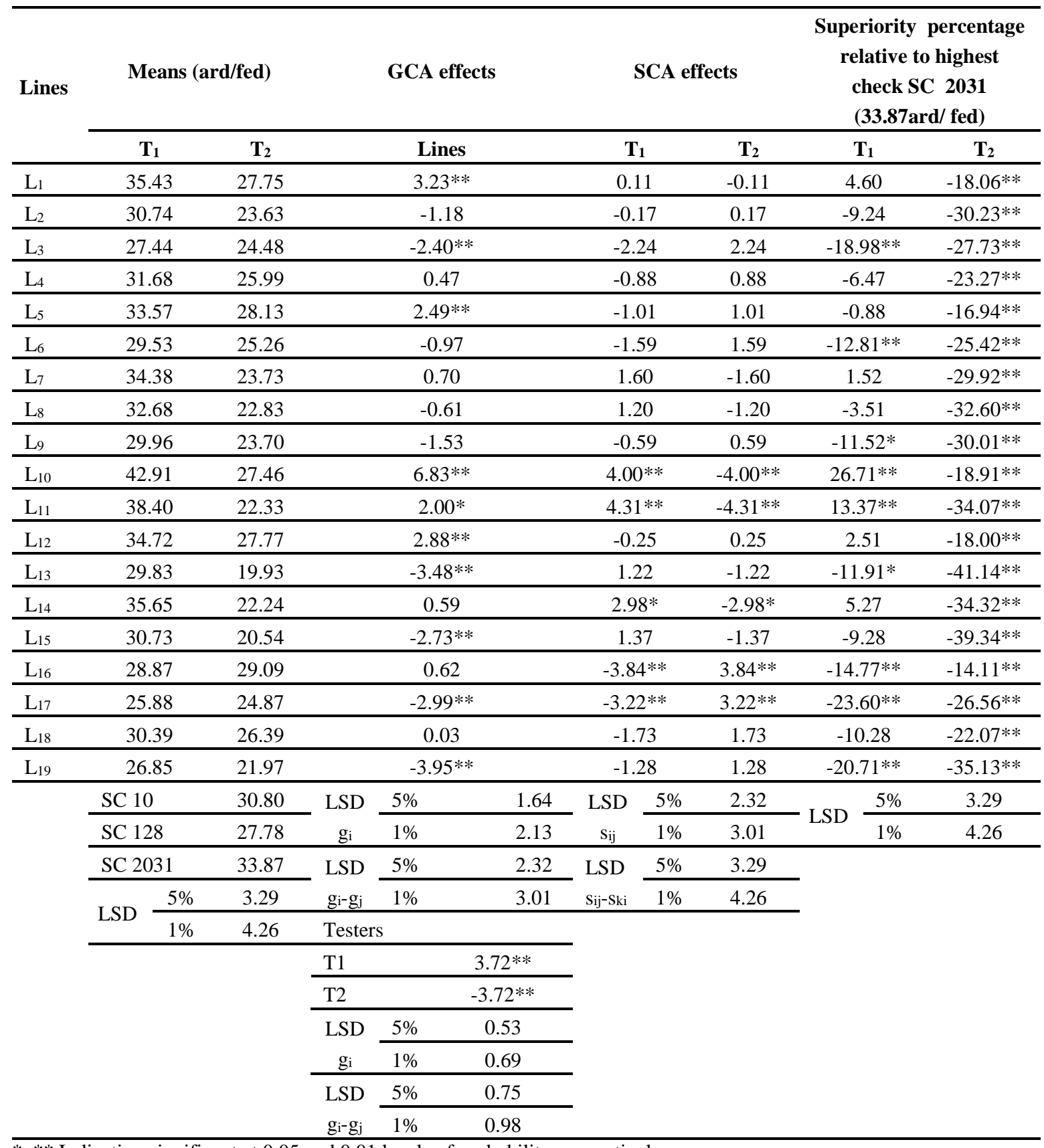

*, ** Indicating significant at 0.05 and 0.01 levels of probability, respectively.

\section{REFERENCES}

Abdallah, T.A.E. (2014). Combining ability estimates using line $\times$ tester analysis to develop high yielding maize hybrids. Egypt. J. Plant Breed. 18(1): $45-55$.

Abd El-Azeem, M.E.M. (2011). Evaluation of some new white maize inbred lines using line $\mathrm{x}$ tester analysis. Egypt. J. Plant Breed. 15(5): 99-112.
Abd EL- Hadi, A.H.; K.S. Kash; A.A El- Shenawy and I.A.I. EL-Gazzar (2009). Evaluation of new yellow inbred lines of maize via line $\mathrm{x}$ tester analysis over two different locations. Alex. Agric. Res., 30:174-187.

Abdel-Moneam, M.A.; M.S. Sultan; S.E. Sadek and M.S. Shalof (2014). Estimation of heterosis and genetic parameters for yield and yield components in maize using the diallel cross method. Asian J. of Crop Sci. Vol. 6 Issue 2, p101. 
Abd El-Mottalb, A.A. (2014). Evaluation of some yellow inbred lines for ombining ability by using topcrosses. Egypt. J. of Appl. Sci., 29 (3) 79-89.

Abd El-Mottalb, A.A.; M.A. Mostafa and H.A.A. Gamea (2013).Combining ability estimates in some white maize inbred lines for yield and other traits. Egypt. J. Plant Breed. 17(3): 13-22.

Abd El-Moula, M.A. (2011). Inheritance of grain yield and other attributes in new yellow maize inbred lines. Assiut J. of Agric. Sci., (Special Issue).

Aboyousef, H.A.; H.A.A. Gamea and Moshera S.E. Sadek (2016). Evaluation of some new white maize top crosses for yield and some other traits. Alex. J. Agric. Sci. 61 (4): 409 - 418.

Abu shosha, A.M. and M.A.F. Habouh (2019). Estimation of combining ability for yield and some (Zeamays,L.) agronomical traits in new yellow maize inbred lines using top crosses method. Minufiya J. Plant Prod.,(4): 121-134.

Alamine, A., M. C. Wali, P. M. Salimath and R. C. Jadeesha (2006). Combining ability and heterosis for grain yield and ear characters in maize. Karanataka J. Agric. Sci. 19: 13-16.

Ali F. I. A. Shah, H. U. Rahman, M. Noor, Durris hahwar, M. Y. Khan, I. Ullah and J. Yan (2012). Heterosis for yield and agronomic attributes in diverse maize germplasm. Australian Journal of crop science 6 (3): 455462.

Al-Naggar, A. M., H. Y. EL-Sherbieney and A. A. Mahmoud (1997). Effectiveness of inbred, single crosses and population as testers for combining ability in maize. Egypt. J. Plant Breed. 1: 35-46.

Aly, A.A. (2004). Combining ability and gene action of new inbred maize lines (Zea mays L.) using line $x$ tester analysis. Egyptian $J$. AppliedSci. 19: 492-518.

Aly, R.S.H. and M.A.G. Khalil (2013). Combining ability for sixteen promising white maize inbred lines for grain yield and yield component traits. Egypt. J. Plant Breed. 17: 143-160.

Aly, R.S.H., E.M.R. Metwali and S. Th.M. Mousa (2011). Combining ability of maize (Zea mays L.) inbred lines for grain yield and some agronomic traits using top cross mating design. Glob. J. Mole. Sci. 6(1): 01-08.

Amin, M. N., M. Amiruzzaman, A. Ahmed and M. R. Ali (2014). Evaluation of inbred lines of maize (Zea mays L.) through line $\times$ tester method. Bangladesh J. Agril. Res. 39 (4): 675683.
Attia, A.N.; M.S. Sultan; M.A. Badawi; M.A. Abdel-Moneam and A.R.M. Al-Rawi (2015). Estimation of combining ability and heterosis for some maize inbred lines and its single crosses. J. Plant Production, Mansoura Univ., Vol. 6 (1): 83-99.

Darrah,L.L., S.A. Eberhart and L.H. Penny (1972). A maize breeding method study in Kenya. Crop Sci. 12: 605-608

Darwich, M.M.B.; Maha G. Balbaa and H.A. Aboyousef (2016). Evaluation of combining ability and type of gene action for grain yield and some related traits in some new yellow inbred lines of maize (Zea mays L.) using top cross. Egypt. J. P. Breed. 20 (4):700 -715 .

Davis, R.L. (1927). Report of the plant breeder. Rep. Puerto Rico Agric. Exp. Sta.. 14-15.

El-Gazzar, I.A.I.; M.A. EL-Ghonemy and S.Th. Mousa (2013). Evaluation of new inbred lines of white maize via line $\mathrm{x}$ tester analysis over three locations.J. Plant Production, Mansoura Univ., Vol. 4 (6): 897 - 906.

El-Gazzar, I.A.I. and M.A.G. Khalil (2012). Combining ability analysis of new yellow maize inbred lines using line $\times$ tester analysis. J. Agric. Res. Kafer El-Sheikh Univ. 38(3): 390-398.

EL-Zeir, F.A.A. (1999). Evaluating some new inbred lines for Combining ability using top crosses in maize (Zea mays L.). Minufiya J. Agric. Res. 24(5): 1609-1620.

Gamea H.A.A. (2015). Estimate of combining ability of new yellow maize inbred lines using top crosses. Egypt J. Agric. Res. 93 (2): 287298.

Gamea H.A.A.; M.M.B. Darwich and H.A. Abo Yousef (2018). Combining ability for some inbred lines in half-Diallel crosses of maize under two different locations conditions. Archives of Agriculture Sciences Journal 1(3):14-25. Special Issue.

Hallauer A.R. and J.B. Miranda (1988). Quantitative Genetics in Maize Breeding. Iowa State, Press, Ames.pp 468.

Horner, E. S; H.W. Lundy; M.C. Lutrick and W.H. Chapman. (1973). Comparison of three methods of recurrent selection in maize. Crop Sci., 13: 485-489.

Ibrahim, M.H.A., M.A. El-Ghonemy and A.A. Abd El-Mottalb. (2012). Evaluation of fifteen yellow maize inbred lines for combining ability by their top crosses. Egypt. J. Plant Breed. 16 (2): 225-236.

Izhar, T. and M. Chakrabory (2013). Combining ability and heterosis for grain yeild and its components in maize inbreds over environments (Zea mays L.) Afr. J. Agric. Res. 8(25):3276-3280. 
Kempthorne, O. (1957). An Introduction to Genetic Statistics. John Wiley and Sons, New York, USA.

Kumar R.; M. Singh; M. Narwal and S. Sharma S (2005). Gene effects for grain yield and its attributes in maize. Natural J. Plant Improvement 7: 105-107.

Mosa, H.E. (1996). Studies on corn breeding. Msc. Thesis, Fac. of Agric. Kafr EL-Sheikh Tanta univ. Egypt.

Mosa, H.E.; A.A. EL-Shenawy and A.A. Motawei (2008). Line $x$ tester analysis for evaluation of new maize inbred lines. J. Agric. Sci.: Mansoura Univ., 33:1-12.

Mosa, H.E. (2010). Estimation of combining ability of maize inbred lines using top crosses mating design. J. Agricultural Research 36: 1-15.
Sedhom, A. S., M. El. M. El-Badawy, A. M. Morsy and A. A. A. El-Hosary (2007). Diallel analysis and relationship between molecular polymorphisms and yellow maize performance. J. Agric. Sci. Benha Univ. 45: 1-20.

Shehata, A.H. (1992). Breeding and genetic studies of maize. Ph.D.Thesis, Agron. Dep., Fac. of Agric. Menofiya Univ., Egypt.

Shehata, A.H. and N.L. Dhawan (1975). Genetic analysis of grain yeild and their crosses. Egypt J. Genetic Cyto. 4: 96-116.

Sprague, G.F. and L.A. Tatum (1942). General vs. specific combining ability in single crosses of corn. J. Amer. Soc. Agron. 34: $923-932$

Steel, R.G.D. and J.H. Torrie (1980). Principles and Procedure of Statistics: A biometrical Approach 2nd. McGraw Hill Book Company. Inc., N.Y., USA.

Umar, U.U.; S.G. Ado; D.A. Aba and S.M. Bugaje (2014). Combining ability, gene action and heterosis in maize (Zea mays L.) under drought stress. International J. of Agric. Innovations and Res. 3(3): 953-958.

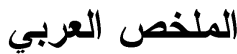

\section{التحليل الوراثي لصفة محصول الحبوب وبعض الصفات المحصولية لبعض سلالات الأرة الثامية البيضاء الجديدة باستخدام تحليل السلالة * الكثاف}

\author{
حمدي السيد احمد جامع \\ مركز البحوث الزر اعية - معهد بحوث المحاصيل الحقلية - قسم بحوث الذرة الثامية
}

\begin{abstract}
نفذت هذه الدر اسة باستخدام احدي و عشرون سلاله مرباه داخليا من الذرة الثامية البيضاء بينهم تسعه عشر سلاله حديثة الاستتباط

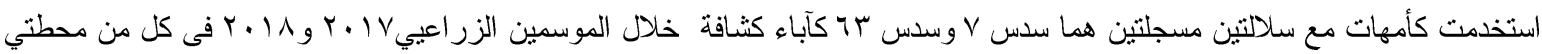

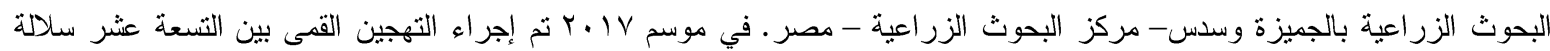

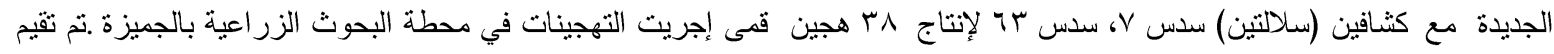

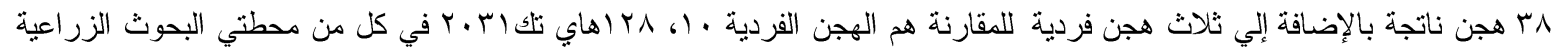

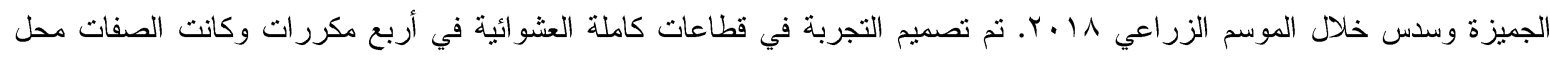
الدر اسة عدد الأيام من الزر اعة حني ظهور ، \% من النورة المؤنثة وطول النبات وارتفاع الكوز ومحصول الحبوب بالإردب للفدان. وكانت أهم النتائج كالتالي: اظهر التباين الراجع إلى المواقع معنوية عالية لجميع الصفات محل الدراسة وكذلك اظهر التباين الراجع للهجن ومكوناتها (السلالة،

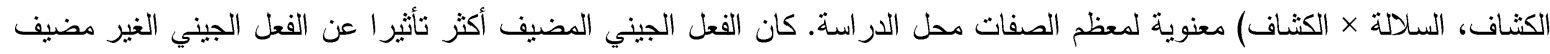

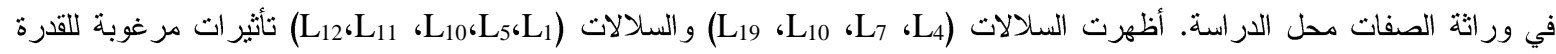
العامة على التالف نحو التبكير فى النضج ومحصول الحبوب على التو الي.

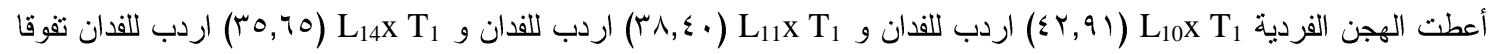
في محصول الحبوب عن أفضل هجن المقارنة وكذلك تأثيرات مرغوبة للقدرة الخاصة علي التالف لصفة محصول الحبوب و عليه يمكن استخدام السلالات الابوية و الهجن المتفوقة الناتجة فى المزيد من الدراسات لاعتمادها كهجن جديدة بعد التأكيد من ثباتها الور اثى.
\end{abstract}

